\title{
Features and Methods of Translation of Long Sentence for Electric Specialty English
}

\author{
Xu Zhang ${ }^{1}$, Liyan Teng ${ }^{2 b}$ \\ ${ }^{1}$ No.169, Changchun Road Jilin City, Jilin province, China \\ ${ }^{2}$ No.169, Changchun Road Jilin City, Jilin province, China \\ a535231128@qq.com, b307532685@qq.com
}

\begin{abstract}
Keywords:Electric Specialty English, Long sentence, Features, Translation Methods.
\end{abstract}
\begin{abstract}
With the rapid development of our country electric power industry and the increasing of international cooperation, translation of electric power English is increasing. But, the translation of long sentence for Electric Specialty English has many problems. Electric Specialty English as a branch of English of science and technology has the features of formal in mode, plain in questions, strong logicality and professional. Electric Specialty English always use long sentences to describe the fact and state the reason in order to make the article rigorous, the information compact; reflect the complex logical relationship between things and phenomena accurately and objectively. This article explores the features of long sentence for Electric Specialty English, translation principles and translation methods.
\end{abstract}

\section{Introduction}

English long sentences generally refers to the sentences which have more than 20 words, sentence structure is arborescence, complicated structure, much modifier, and Chinese short, there is a big difference between line structure and short of the Chinese sentences. In order to do translation accurately, we must understand the characteristics of English long sentences, carefully analyze the sentence structure, and rely on a certain translation skills, so that the translation can express the original meaning accurately, and with the Chinese expression habit.

Translation of Electric Specialty English has certain difficulty, there are many long sentences in it, and there are differences between the way of English and Chinese expression. Thus translation of long sentence for Electric Specialty English increases the difficulty to a certain extent. This article explores the features of long sentence for Electric Specialty English, translation principles and translation methods.

Electric Specialty English is different to daily English, literature English and its features mainly include professional vocabulary, more passive voice, and long sentences.

Professional vocabulary. Power professional vocabulary refers to that words have special significance in the electric power industry, usually the words also have common sense, such as "current" in daily English, it has the meaning of "now, recently, circulating", and "current" in the electric power industry it means "electricity".

More passive voice. The use of passive voice in English is very wide, especially in electric English, you can find passive voice in almost everywhere, in electric power English, often put the name of the object as the subject in the prominent position, describe the action or state by the passive voice. In Chinese the passive voice be used less, a lot of passive actions often make use of 
the passive significance words or he active voice for expressing.

Such as: "Transformers are sometimes overloaded as users install more and more appliances"[1]

Long sentences.There are some long sentences in Electric Specialty English, to make a more clear logical relations.

Such as "The generators, lines, and other equipment which form an electric system are arranged depending on the manner in which loads grows in the area and may be rearranged from time to time."

In this sentence, "which” guides the object clauses layouts the logical relation well, an electric system was made of generator, lines, and other equipment.

\section{Features of long sentence for Electric Specialty English}

Sentence structure is complex. Due to the large amount of information to express scientific facts or phenomena, electric professional papers always need to use a long sentence which can fully express complex meanings, long sentences do expand on the basis of the basic organization, add all kinds of modifiers such as attributive, adverbial, use compound sentence or compound element, such as double predicate, and object,attributive to increase the parenthesis, appositive or use inversion and ellipsis structures. Often parallel structure, clause, crisscross use a predicate structure, make the sentence becomes complicated; sometimes in a sentence at the same time appear many clauses, multi-layer parallel structure.

Such as: Instruments that continuously monitor current, voltage, and other quantities must be able to identify the faulted equipment and to bring about operation of the circuit breakers, which remove it from service, while leaving in service all other equipment on the operating system.

There are 42 words in this sentence, the structure is complex, the subject with a "that" after the "instruments" leading the attributive clause; the predicate part of complex structure. "To identify..." With "to" bringing about..." As "be able" after the paratactic structure; after the noun "circuit breakers" is an attributive clause. End of the sentence "while leaving in service all other equipment on the operating system" is a company adverbial participle mechanism, including "all other equipment on the operating system" is the object for "leaving", in order to make the sentence balance and make the object move backward.

Modifiers complex.Due to scientific, using complex postpositive attributive or appositive to explain some concepts or phenomena, also is one of the major English characteristic. Modifiers of electric power professional English long sentences are long and complicated, and sometimes even a set of one in one or one with few situations. Modifiers can be clauses, the predicate structure, the preposition structure, etc.

Such as : Some metals are better conductors of electricity than others, which means that the former have atoms that contain more free electrons than the latter.

This sentence used "which" leading a non-restrictive clause, for supplement. And the object of the verbs in the attributive clause is a clause leading by "that". There is another attributive clause in the object clause that is "atoms "after" that contain more free electrons than the latter". Inside of the attributive clause organization is very complex.

Moreparatactic structure.There is more paratactic structure in long sentence for Electric Specialty English. It would be parallel in word, phrase, and structure of the verb or parallel subordinate clause. Sometimes the paratactic structures are different, for example, Sometimes the paratactic composition structure is different, such as prepositional phrase and the attributive clause can act as attributive, and paratactic structure will sometimes be omitted, all of that have made the 
paratactic structure be complicated. So first, clarify the relationship between the compound is of great importance to translation.

Such as :This system is economical as it flattens out the variations in the load on the power grid, permitting thermal power stations such as coal-fired plants and nuclear power plants that provide base-load electricity to continue operating at their most efficient capacity, while reducing the need to build special power plants which run only at peak demand times using more costly generation methods. [3]

The backbone of the sentence is clear, as a system table structure, guided by "as "adverbial clauses of reason complex structure. In this clause has two segmentation structure side by side adverbial of result, namely "permitting thermal power...capacity "and" reducing...methods ", two parallel between the adverbial" while "link, the table shows correlation. The structure of the two coordinate adverbial is very complicated, and all of them have the subordinate clause.

Such as: The direction of current flow may be show either by a hollow arrowhead or by supplying the current symbol with a double subscript whose first digit identifies the unction at a higher potential and the second the junction at a lower potential.

In this sentence, "a double subscript" behind the "whose" to guide the attributive clause, the attributive clause consists of two parallel sentence composition, in the middle of "and" link, two patterns of clauses are the same, the second clause undertook earlier, omitting the with the first clause of the same part of "the second the junction at a lower potential=the second digit identifies the junction at a lower potential."

Specialty. The other important feature of long sentence for Electric Specialty English is strong specialty, the conventional expression in electric power field is an important fact that cannot be ignored.

Such as: Whichever way the two two-way switches are left , one of the wires is“alive” and the other is dead.

The "alive" and "dead" in this sentence is "alive" and "dead" in the field of electric power, with electronic and non-electronic means. Many specialized words are derived from the basic meaning of ordinary words, so the translator must have professional background knowledge, understand the power professional vocabulary.

Such as : Ideal voltage and current source are active circuit elements, while resistances and conductance are passive elements.

In this sentence, "voltage ,current source ,active circuit element ,resistance ,conductance ,passive element” is a term of electric power, with a very clear power professional.

\section{Principle of translation of long sentence for Electric Specialty English}

Translation of long sentence for Electric Specialty English requires fluent, concise and logical based in convey information accurately, which can also be regarded as principles for the translation of Science and Technology English.

Accurate expression. Accurate expression is the requirement of the translator in the translation of the original content of the full and accurate expression of the original. English translation of science and technology is an important way for us to learn the foreign advanced technology, as a 
member of the CRS wind group of overseas department, the writer is deeply appreciated the importance of accurately in the expression of long sentences in scientific English translation. As a result of a lot of scientific and technical information and data parameters translation is to be used as our research and practice of the operation of the document to use, and its translation must be rigorous and accurate. Any errors or deviations in expression may mislead the scientific research as well as the complete failure of the practice operation. For example, the power professional English "design resistances the" and "characteristic strength the" means"设计阻抗"'特征强度", if the translator does not have a solid professional knowledge, it is possible to translate it: and and other non-professional words, so that readers have a bias in understanding.

In addition, the translator needs to determine the meaning according to the context in the translation of the meaning of a word. As long as it is translated, the translation can be correctly obtained. Long sentences and professional vocabulary, in scientific English, such as the power of professional English in "feathering position" should be translated torather than "brisk" and "pitch system for variable rather than, therefore, the translator should have a solid professional knowledge. In sentences, complex long sentences in scientific English structure, more clauses and noun structure, the translator should understand the original, distinguish meaning group and logical relationship, so that accurate expression of the translated text.

Smooth and fluent. Fluent language must comply with the requirements of good habits and expression of Chinese grammar structure. Just do the accurate expression of the original information is not enough, but also to ensure that the appropriate choice of words in translation to punctuate, sentence is smooth, in line with the target language grammar rules and habits of expression. Scientific English used to express the complex concept of long sentences, while the Chinese are accustomed to using the phrase as a structured narrative. In translation, the translator should avoid being bound by the syntactic structure of the source text, and adopt appropriate translation techniques according to the Chinese language, try to make the translation smooth, let the reader feel translationese, no more stiff obscure phenomenon and illogical and ungrammatical.

Such as : This Section applies for wind turbines in which one breaking system is a mechanical rotor break and the other breaking system is a blade-pitch system insofar as the blade-pitch system is so constructed that when a single component breakdown, it can prevent the pitching of all rotor blades.

Translator in the translation of the attributive clause in this sentence, if in accordance with the original structure placed in front of it modified the "generator", will the translation for "this section content is suitable for using a mechanical wind wheel brake and a blade pitch system as the braking system of wind turbine , ... , ",long attributive, translationese is overweight, readers when reading will feel stiff obscure, the author here will translation of attributive clause into two phrases, placed behind the "wind turbines" to defined and described, make the translation more smooth.

Strong logicality.Strong logicality is one of the features of EST (English for science and technology), the English of science and technology are mainly in logic of statements describing the process, and so the translator in translation not only to express accurately, but also to grasp the logic of the original, making the translation read rigorous logic is reasonable. Similarly, in the long sentences in EST translation, it is necessary to highlight the logical features of long sentences in scientific English, organized the long sentences according to certain logical relationship this requires the translator to deconstruction and reconstruction of the original sentence to proper express the logical connotation and focus of the text. 
The long sentences in scientific English translation principles, can effectively guide the English of science and technology, especially the translation of long sentences in English of science and technology, under the guidance of the principle, the translator can according to the actual situation flexibly using sequential translation, reverse translation, division translation to translate long sentences in scientific English.

\section{Common methods of translation}

For the translation of English long sentences, the first thing is to carefully analyze the syntactic structure, an accurate understanding of sentence meaning, then adopt flexible translation methods for translation, the commonly used methods of translation are: sequential translation, reverse translation, division translation, embedded method and restructuring method.

Sequential translation.Although there are many differences between the two languages of English and Chinese, there still are a lot of common in people's thinking. In the expression of a series of things, the expression of English and Chinese are similar, so, the translation can be used in the sequential translation method, that is, in the original order of the English sentence.

For example, to understand how diodes and other semiconductor devices can do what they do, it is first necessary to understand the basic structure of semiconductor devices.

There are 27 words in this sentence; the sentence is a standard long sentence. The sentence is not set phrase to do sentence adverbial, the structure of the adverbial is also set to have a "how" to guide the object clause, Object clause object is a "what" to guide the noun clause, the clause is a subject of post sentence, "it" is formal subject, the real subject is infinitive subject "to understand the basic structure of semiconductor devices". This sentence is complex, but has similarity of word order in Chinese, so it can use sequential translation method directly translated.

Reverse translation. Anglo Americans pay attention to logic, often first to express judgments or conclusions and then show the background, causes, conditions, etc. Therefore, in English sentences, the conclusion is generally in the first sentence, the condition, the reason, the method and so on are put in the back, while Chinese people like to describe the natural order in accordance with the occurrence and development of things, so Chinese sentences are often followed by the results from the facts to the conclusion, from the conditions to the consequences of the order. Due to the difference of two kinds of word order, in this kind of English long sentences translation, often require the original order is reversed over, according to Chinese expression habit from the organization of a new language and translation. That is the reverse translation.

Such as: A series circuit is formed when a number of resistors are connected end -to-end so that there is only one path for current to flow. [5]

The clause in this sentence "a number of resistors when...... to flow "appears after the" a series circuit is formed in the main clause, so in the translation will be the order is reversed over.

Division translation.Some English sentences logic sometimes translated into a perplexing, Chinese sentence would be lengthy or unclear semantics. This often take a piecemeal approach, the original sentence in the words of the junction between the disconnection, or in accordance with the sense group division, were translated into several independent Chinese phrases. This method is called division translation.

For example: As we can see from Fig.27.1 three or more levels of control devices, each with distinct duties form a computer system hierarchy, where the upper level computer depend on lower level devices for process data, and the lower level system in turn depend on the higherLevel systems for ever more sophisticated control functions, such as overall plant optimization.

The sentence structure is more complex, the first sentence is "as" to guide the non-restrictive 
attributive clause and the main clause subject and predicate has a parenthesis, the latter part of a sentence "while" to guide the clause attributive, modified "hierarchy", the translation will be non-defining attributive clause and the main clause disconnect alone translated into a sentence, as to avoid due to the long sentences and make the semantic is not clear, in line with the characteristics of Chinese language concise.

Embedded method.English modifier is often put on the back, and the modifier proposed is a Chinese expression habit, so in the translation into Chinese, often need to move the English language of the modifier to the front of the center word, this method is embedded in the law. This approach is mainly used to translate the attributive structure, and sometimes also used to translate some adverbial structure.

Such as: They will always be attracted from a sense having an excess of electrons , thus having a negative charge , to a source having a deficiency of electrons which has a positive charge.

The structure of the two words in this sentence "having an excess of electrons, thus having a negative charge" and "having a deficiency of electrons which has a positive charge" are respectively used as post modifier, which modifies the previous term. They are translated into "of...... "Structure is in the front of the center of the word, in line with the expression of Chinese habits.

Restructuring method.The restructuring method refers to the translator's understanding of the meaning of the original sentence, breaking the order structure of the original sentence, and translating the meaning of the original sentence according to the Chinese expression habit. This method used for complex structure translation of long sentences, the translated text jumped out of the shackles of the original sentence structure, fully in accordance with the Chinese habit of re expression of the original meaning. This method is one of the most difficult translation methods to grasp.

Such as: An effective dynamic control of each operating unit of the plant to assure that it is operating at its maximum efficiently of production capability, product quality and /or of energy and materials utilization based on the production level set by the scheduling and supervisory functions listed below.

This sentence has a number of subordinate clauses and coordinates structure, and the logic relation is complex. After clarifying the logical relationship of the original English sentences, the translator has to re integrate the whole sentence, and then in accordance with the Chinese logic expressions, translated into fluent but faithful Chinese sentences.

\section{Conclusion}

Translators should grasp the syntactic and professional characteristics of long sentences in the power of professional English, according to the wealth of English vocabulary, grammar knowledge and the power of professional knowledge, to reconstruct the original text and to make the translation correct and accurate transmission the original information, while in line with the Chinese language habits, so that the translation can be natural and fluent.

\section{References:}

[1].H.M. Duan.The Theory of Translation of Long Sentences in Scientific English(In Chinese)[J],Science and Technology Information Development and Economy,2004,(7). 
[2]..Fang M.Z. Fang. Translation Studies and Practice(In Chinese)[M].Qingdao:Qingdao Press 1999.

[3]. X.Y. He Comprehensive Case Teaching of Translation Theory (In Chinese) [M].Beijing:Renmin University of China Press 2010.

[4]. Z.L.Huang. Translation Methodology(In Chinese) [M].Beijing:China Social Sciences Publishing Press,2009.

[5]. R.B. Liu, Electric Power Professional Knowledge (In Chinese) [M].Beijing:China Electric Power Press,2006. 